\title{
"EL DERECHO AMBIENTAL ENTRE LA CIENCIA, LA ECONOMÍA Y LA SOCIOLOGÍA: REFLEXIONES INTRODUCTORIAS SOBRE EL VALOR NORMATIVO DE LOS CONCEPTOS EXTRAJURÍDICOS"
}

\section{ENVIRONMENTAL LAW IN SCIENCE, ECONOMICS AND SOCIOLOGY: PRELIMINARY REFLECTIONS ON THE REGULATORY VALUE OF EXTRALEGAL CONCEPTS.}

\author{
SANTiago M. Álvarez Carreño \\ Profesor Titular de Derecho Administrativo \\ Universidad de Murcia \\ santialv@um.es
}

\begin{abstract}
"De acuerdo con las tesis aquí sustentadas, para que el hombre pueda garantizar su pervivencia futura, debe dotarse de un cuerpo normativo que recoja los dictados de las Ciencias de la Naturaleza, para una prudente utilización económica de la Biosfera. Para ello se necesitará contar además con el apoyo de la Ética y de la Física"

Ramón MARTín MATEO, La revolución ambiental pendiente, 1999
\end{abstract}

RESUMEN: El presente trabajo reflexiona, a modo de introducción general a las aportaciones monográficas que componen el monográfico, sobre la dependencia del Derecho ambiental respecto de conceptos provenientes de las ciencias naturales (física, química, biología, ecología...) y sociales (economía, sociología...) como modo de aproximación tanto a la grandeza de su objeto de estudio como, a la vez, a su situación debilitada en un mundo de incertidumbres que son utilizadas en muchas ocasiones además por los sectores interesados para evitar o minimizar una aplicación eficaz de la norma ambiental. 
ABSTRACT: The present academic paper tries to reflect, as a way of general introduction to the monographic contributions that make up the present volume, the dependence of environmental law on concepts coming from the "hard" sciences (physics, chemistry, biology, ecology ...) and from social sciences (economics, sociology ...). This particular situation of this branch of our legal system allows us to observe both the greatness of its object of study and, at the same time, its weak position in a world of uncertainties, often used by interested stakeholders to avoid or minimize the effective application of the environmental rules.

RESUM: Aquest treball reflexiona, a tall d'introducció general a les aportacions monogràfiques que componen el monogràfic, sobre la dependència del dret ambiental respecte de conceptes provinents de les ciències naturals (física, química, biologia, ecologia...) i socials (economia, sociologia...), com a aproximació tant a la grandesa del seu objecte d'estudi com, alhora, a la seva situació afeblida en un món d'incerteses, que són utilitzades en moltes ocasions a més pels sectors interessats per evitar o minimitzar una aplicació eficaç de la norma ambiental.

PALABRAS CLAVE: Derecho ambiental - Conceptos científicos - Riesgo — Cambio climático - Diálogo interdisciplinar

PARAULES CLAU: Dret ambiental - Conceptes científics - Risc - Canvi climàtic — Diàleg interdisciplinari

KEY WORDS: Environmental Law - Scientific concepts - Risk - Climate Change - Interdisciplinary dialogue

\section{SUMARIO:}

I. La dependencia de la evolución científica y tecnológica como característica especialmente destacable del Derecho ambiental como rama jurídica. II. La posición de la ciencia en el núcleo esencial de la norma ambiental. III. El intento de agrupación de los conceptos en base a su diferente origen y finalidad. IV. La incertidumbre científica como fuente de debilidad del Derecho ambiental: su reflejo en la jurisprudencia constitucional española. V. Final: la necesidad de un diálogo fluido y fructífero entre los diferentes saberes que confluyen en la determinación del contenido de las normas ambientales. Bibliografía. 


\section{LA DEPENDENCIA DE LA EVOLUCIÓN CIENTÍFICA Y TECNOLÓGICA COMO CARACTERÍSTICA ESPECIALMENTE DESTACABLE DEL DERECHO AMBIENTAL COMO RAMA JURÍDICA}

La profunda preocupación por el estado en que se encuentra el medio ambiente representa un "signo de nuestro tiempo". El grado de desarrollo económico, industrial y tecnológico alcanzado ha producido una degradación tan acusada de los recursos naturales que este problema se ha convertido en uno de los desafíos esenciales que la humanidad tiene que resolver. Existen, sin embargo, como es notorio, diversas posturas a la hora de valorar el verdadero alcance del problema ambiental. Para unos, la situación no es tan grave: al fin y al cabo, a lo largo de la historia de nuestro planeta ya se han producido catástrofes ambientales que tuvieron su origen en causas naturales. Se confía, además, en una suerte de «optimismo científico y tecnológico» de modo que el propio hombre -agente principal de la actual crisis ambiental- será capaz de resolverlo con ayuda de los avances que se van produciendo en esos ámbitos. Esta línea de pensamiento acusa a aquellos que no comparten este optimismo de "catastrofismo", "extremismo" o, incluso, de dejarse llevar por una "moda ecologista". Frente a ello, con diversos matices, se encuentran quienes señalan la gravedad de la crisis ambiental y reclaman cambios más enérgicos en las actuales políticas económicas y energéticas que permitan modificar el rumbo para transitar hacia una sociedad con mayor armonía ambiental o ecológica. Desde este segundo punto de vista, se estaría produciendo un auténtico cambio de paradigma cultural y social al que acompaña una creciente actitud ciudadana más concienciada y exigente frente a los poderes políticos.

El Derecho ambiental traduce en normas y principios este especial momento evolutivo y precisa para su correcta interpretación y aplicación, dado el alcance y trascendencia de la grave problemática a la que se enfrenta, del adecuado manejo de conceptos provenientes de otras ciencias. Como señala MEYNER, "el Derecho concibe su objeto gracias a instrumentos conceptuales directamente ligados a la realidad medioambiental. Sin embargo, los conceptos implican una generalización y una abstracción de los hechos, es decir, una cierta recreación de la realidad. Esta realidad está contenida en los conceptos que, además de designar una cosa, transmiten valores y una comprensión determinada de la 
realidad de modo que el Derecho pueda actuar sobre ella. Los conceptos descriptivos conforman de este modo una realidad jurídica autónoma, propia del Derecho ambiental"1.

Esta dependencia de la evolución científica y tecnológica, como característica especialmente destacable de esta rama jurídica, ya ha sido señalada entre nosotros por voces tan autorizadas como MARTín MATEO², ESTEVE PARDO³, PAREJO ALFONSO ${ }^{4}$, BETANCOR $^{5}$ y, de las que se han utilizado para la elaboración

\footnotetext{
* Este trabajo forma parte de los resultados de la estancia de investigación en la Universidad La Sorbona que, bajo la dirección de la Profesora Marta TORRE-SCHAUB, realicé durante los meses de marzo a mayo de 2019 (ref. 20589/EE/18) financiada por la Fundación Séneca-Agencia de Ciencia y Tecnología de la Región de Murcia con cargo al Programa Regional de Movilidad, Colaboración e Intercambio de Conocimiento "Jiménez de la Espada"».
}

1 A. MEYNER (2014), "Le rôle des concepts dans la fondamentalisation du droit de l'environnement", en P. MILON / D. SAMSON, Révolution juridique, révolution scientfique. Vers une fondamentalisation du droit de l'environnement?, Presses Universitaires d'Aix-Marseille (PUAM), Aix-en-Provence, pp. 118.

Frente a esos conceptos denominados descriptivos, la profesora MEYNER detecta en el Derecho ambiental la existencia de conceptos prescriptivos a través de los cuales se condensan los objetivos propios de esta rama jurídica: "algunos de ellos constituyen orientaciones más o menos imperativas como los conceptos de "generaciones futuras", "desarrollo sostenible», «interés general», «estado de conservación favorable», «medio ambiente sano». Otros son portadores de principios que conforman toda la materia, como el de precaución, compensación o el de equilibrio" (Ibídem, p. 120).

2 Vid. a modo de ejemplo su último artículo publicado Ramón MARTín MATEO (2017), "La revolución ambiental pendiente", Revista Aranzadi de Derecho Ambiental, núm. 38, pp. 21-41. La incansable curiosidad intelectual del Maestro impregnó toda su obra. Así, en su libro La verde energía de la biomasa (Thomson-Civitas, 2008) apuesta de forma decidida por destacar las nuevas posibilidades que ofrecen los avances tecnológicos que permiten la expansión del uso de esta nueva fuente de energía. En su breve artículo "El avance científico: nuevos retos para el derecho sanitario" (DS -Derecho y salud, vol. 9, núm. 1, enero-junio 2001, pp. 21-26) reflexiona sobre la recepción por el Derecho de los avances científicos. No me resisto a transcribir las palabras con que cierra este breve estudio que esperemos no fueran proféticas: "Suelo afirmar que una de las causas que nos ha permitido llegar hasta aquí es el que, durante la última guerra mundial, o más bien durante la Segunda Posguerra mundial, a uno u otro lado del teléfono rojo no había un loco. Si un insensato hubiera dado la orden de activar el dispositivo adecuado, hubiéramos tenido posiblemente un invierno nuclear que también nos hubiera puesto como especie en entredicho".

${ }^{3}$ Esenciales en esta materia resultan sus ya clásicas monografías José ESTEVE PARDO (1999), Técnica, riesgo y derecho. Tratamiento del riesgo tecnológico en el derecho ambiental y (2009) El desconcierto de Leviatán. Política y derecho ante las incertidumbres de la ciencia, Marcial Pons, Madrid.

Muchas de las reflexiones e ideas que se tratan de exponer en este estudio introductorio son debidas a la influencia y conocimientos aportados por la lectura de esas y otras seminales contribuciones de este magistral autor.

4 Luciano PAREJO ALFONSO (coord.) (2015), El derecho ante la innovación y los riesgos derivados del cambio climático, Tirant lo Blanch, Valencia.

${ }^{5}$ Andrés Betancor Rodríguez (2014), Derecho ambiental, La Ley, Madrid. 
de estas notas y con disculpas por si se incurriera en un más que probable olvido de algún otro autor destacado o de alguna referencia igualmente significativa, LOZANO 6 .

\section{LA POSICIÓN DE LA CIENCIA EN EL NÚCLEO ESENCIAL DE LA NORMA} AMBIENTAL

Resulta un hecho constatable que la política y legislación ambiental no han adquirido la importancia que tienen en la actualidad hasta que la comunidad científica ha expresado de modo rotundo su preocupación por la alarmante degradación continua de las condiciones ambientales. La ciencia se encuentra de este modo en el corazón mismo de la norma ambiental con la cual mantiene unas relaciones dinámicas y complejas que no se limitan a una simple transcripción normativa. Como afirman BORN y DE SADELEER, "se distinguen las ciencias fundadoras del Derecho ambiental partiendo de la distinción, ya clásica dentro del mismo, entre un derecho de protección contra las emisiones y la contaminación («nuisances»), por una parte, y de otra un derecho de la protección de la naturaleza. El primero nace de la legislación higienista del siglo

Sobre la adaptación del concepto «mercado» para su puesta en servicio en favor de la protección del medio ambiente vid. su presentación de la sección monográfica "Nuevas tendencias en el Derecho ambiental", Revista General de Derecho Administrativo, núm. 25, octubre 2010. En concreto, BETANCOR reflexiona sobre la introducción del instrumento de mercado, en su opinión, una opción razonable, pero señalando que se trata en todo caso de un mercado "artificial" pues el título objeto de negociación es un título jurídico administrativo, creado por una decisión de la Administración, como sucede con el mercado de derechos de emisión (Ibídem).

Sobre los cambios en la regulación jurídica que traen causa en avances del conocimiento científico y del progreso tecnológico en relación específica al agua vid. Santiago M. ÁlvarEZ CARREÑO, "Los derechos privados sobre el agua en países de common law: reflexiones sobre su evolución y adaptación a las necesidades de cambio", en Ibídem.

${ }^{6}$ Blanca LOZANO CUTANDA (2016), "Derecho ambiental: algunas reflexiones desde el Derecho administrativo", Revista de Administración Pública, núm. 200, pp. 409-438.

Son muchas las reflexiones que sobre la dependencia de la evolución científica y técnica hace la profesora LOZANO en este artículo (la necesaria base científica para que sean admisibles las medidas de naturaleza regresivas, los nuevos desarrollos tecnológicos que plantean la necesidad de respuesta jurídica...). En concreto, sobre los problemas que amenazan al ecosistema de la Tierra, afirma que "La sociedad utiliza el derecho para dar una respuesta a los retos y dilemas de cada tiempo histórico. El problema que se aborda con el derecho ambiental es, a decir de los científicos, el de la desestabilización antrópica de la biosfera: como resultado de las fuerzas motrices activadas por la revolución industrial (crecimiento económico, explosión demográfica -en el siglo XX la población humana se triplicó y los demógrafos de Naciones Unidas prevén que alcance los 9.600 millones de personas a finales del presente siglo, uso masivo de las energías fósiles, consumo de masas-), una infinidad de precisiones e impactos ambientales con efecto acumulativo y sinérgico han provocado la desestabilización de las funciones de soporte de la biosfera, y la comunidad científica insiste en que existe el riesgo cierto de un cambio irreversible" (p. 410). 
XIX y encuentra su fundamento inicialmente en la química para estar impregnado hoy por multitud de disciplinas científicas "duras», como la física y la química. La otra rama, influida en sus orígenes por una concepción casi romántica de la naturaleza, comenzó seguidamente y de manera progresiva a estar inspirada por las ciencias llamadas «naturales» y por la agronomía, después por la ecología, ciencia sobre la que se basa en la actualidad el derecho del medio ambiente, en lo que afecta a las relaciones complejas de los seres vivos con su medio"

Esta estrecha vinculación entre el Derecho ambiental y las diferentes ciencias en las que se apoya obliga a afrontar determinados desafíos relacionados con las grandes diferencias entre objetivos y finalidades, marcos temporales de desarrollo y exigencias de verificabilidad y prueba de sus resultados en los ámbitos científicos en comparación con el jurídico ${ }^{8}$. En muchas ocasiones será necesario recurrir a una traducción jurídica de conceptos y resultados científicos para lograr la aplicabilidad en el mundo del Derecho de los conceptos científicos que se han incorporado a la norma ambiental ${ }^{9}$. Por otra parte, muchos de los

\footnotetext{
7 Vid. CH.-H. Born / N. De SADELEER (2001), nota bibliográfica aparecida en Revue Juridique de l'Environnement (núm. 3, pp. 555-557) al libro de E. NAIM-GESBERT, Les dimensions scientifiques du droit de l'environnement -Contribution à l'étude des rapports de la science et du droit, Tesis doctoral, Universidad de Lyon, 1997 [énfasis añadido].
}

${ }^{8}$ Sobre esta cuestión vid. José ESTEVE PARDO (2003), "Ciencia y Derecho ante los riesgos para la salud. Evaluación, decisión y gestión”, Documentación Administrativa, núm. 265-266, pp. 137149.

${ }^{9}$ WEIS nos recuerda que para ayudar a traducir las certezas científicas a los entes decisorios los órganos consultivos como el Panel Intergubernamental sobre Cambio Climático ha usado escalas que relacionan las probabilidades basadas en el teorema de BAYES con sus equivalentes formulaciones legales como "más allá de una duda razonable" o "base razonable para afirmar ..." (cito por J. W. MOORE / L. NOWLAN / M. OLSZYNSKI, / A. L. JACOB, / B. FAVARO / L. COLLINS / GLT.-L. WILLIAMS DAVIDSON / J. WEITZ (2018), "Towards linking environmental law and science", FACETS -a multidisciplinary open access science journal, vol. 3, pp. 375-391).

Esta llamada a la interdisciplinariedad resulta esencial para enfrentar los nuevos desafíos que nos plantean los avances en el conocimiento científico. Vid. D. OWEN / C. NOBLET (2015), "Interdisciplinary Research and Environmental Law", Ecology Law Quaterly, vol. 41, Issue 4, pp. 887-938.

Por otra parte, los prejuicios epistemológicos -no exentos en ocasiones incluso de componentes netamente políticos- que subyacen a la marginación por las propias instituciones académicas y, sobre todo, por las agencias de evaluación de la calidad de la investigación de los esfuerzos emprendidos por aquellos investigadores que intentan poner la ciencia al servicio del desarrollo sostenible y que, con esta finalidad, traspasan los límites de sus respectivas disciplinas vienen a su vez determinados por la inexistencia de criterios adecuados para valorar adecuadamente este esfuerzo investigador. Sobre la necesidad de una validación de la sostenibilidad de la actividad científica e investigadora que, por su parte, debe contribuir a convertir a los propios científicos en agentes del cambio de conciencia en sentido ambiental en A. KLÄY / A. B. ZIMMERMANN / F. SCHNEIDER (2015), "Rethinking science for sustainable development: Reflexive interaction for a paradigm transformation", Futures, vol. 65, pp. 72-85. 
conceptos y principios utilizados en el Derecho ambiental tienen tanto un componente científico como valorativo. En esos casos, se debe tender a diferenciar las funciones del científico de las del jurista que debe más bien atender a ese elemento de ética social ${ }^{10}$.

Sucede así de manera paradigmática con el «principio de precaución» de acuerdo con el cual recae en el ámbito de lo jurídico la decisión sobre el nivel de riesgo que resulta tolerable para una sociedad en un momento concreto ${ }^{11}$. Los recelos expresados por determinados sectores de la industria llevan a intentar configurar un alternativo "principio de innovación" que vendría a contrapesar los supuestos frenos que a la innovación tecnológica y al avance del conocimiento podría suponer una interpretación amplia del principio de precaución ${ }^{12}$. Del mismo modo, la identificación y delimitación del concepto clave para la legislación sobre protección de la naturaleza y la biodiversidad de «ecosistema» ${ }^{13}$ como entidad no parece tan clara para los científicos. Ahora

\footnotetext{
10 Un concepto que viene en nuestra ayuda para intentar conjugar este necesario dialogo entre ciencias y saberes muy diferentes $y$, a la vez, permitir la toma de decisiones en contextos fuertemente participativos es el «internormatividad» elaborado por CARBONNIER para tratar de explicar la relación entre derecho y religión y derecho y moral pero de aplicación creciente al campo ambiental. Cfr. A. POMADE (2012), "Penser l'interdisciplinarité par l'internormativité. Illustration en droit de l'environnement", Revue interdisciplinaire d'études juridiques, 2012/1, vol. 68, pp. 85-106.

11 El principio de precaución no tiene, sin embargo, aplicación exclusiva en el ámbito del medio ambiente sino que su ámbito es mucho más amplio y abarca aquellos casos en los que los datos científicos son insuficientes, no concluyentes o inciertos, pero en los que una evaluación científica objetiva preliminar hace sospechar que existen motivos razonables para temer que los efectos potencialmente peligrosos para el medio ambiente y la salud humana, animal o vegetal pudieran ser incompatibles con el alto nivel de protección que exige el ordenamiento jurídico vigente. Vid. César CIERCO SEIRA (2004), "El principio de precaución: reflexiones sobre su contenido y alcance en los derechos comunitario y español", Revista de Administración Pública, núm. 163, pp. 73126; Gabriel DOMÉNECH PASCUAL (2006), Derechos fundamentales y riesgos tecnológicos: el derecho del ciudadano a ser protegido por los poderes públicos, Centro de Estudios Políticos y Constitucionales, Madrid; Antonio E. EMBID TELLO (2009), "El principio de precaución", en José Antonio SANTAMARÍA PASTOR (dir.), Los principios jurídicos del Derecho Administrativo, La Ley, Madrid; y, últimamente, Blanca SORO MATEO (2017), "Construyendo el principio de precaución", Revista Aragonesa de Administración Pública, núm. 49-50, pp. 87-151.
}

12 La articulación de este principio en el sitio web del EUROPEAN RISK FORUM $<$ http://www.riskforum.eu/> [Último acceso: 17/04/2019]. El intento de anclar este principio en el Derecho de la UE se ha diluido ante la emergencia del "principio de no regresión". Vid. infra esta misma Introducción.

${ }^{13}$ Ley 42/2007, de 13 de diciembre, del Patrimonio Natural y de la Biodiversidad define en su art. 3, núm. 10, "ecosistema» como "complejo dinámico de comunidades vegetales, animales y de microorganismos y su medio no viviente que interactúan como una unidad funcional".

Entre los principios que inspiran esta ley (art. 2) aparece repetidamente citado. Así, en sus letras a) ("El mantenimiento de los procesos ecológicos esenciales y de los sistemas vitales básicos, respaldando los servicios de los ecosistemas para el bienestar humano..."), c) (“La utilización 
bien, si se tiene en cuenta la dimensión de "ecosistema» como modelo que utiliza la ecología para investigar cómo funciona el mundo natural entonces adquiere otro significado al convertirse en una condición definida por el propio investigador, donde resulta ya igualmente válida su visión como concepto netamente funcional o como unidad con extensión espacial ${ }^{14}$. La ciencia debe proporcionar el modelo espacial que permita, seleccionando las variables más relevantes y las escalas adecuadas para la aplicación deseada, identificar las unidades geográficas que representen la funcionalidad de los ecosistemas. Esta función de simplificación resulta, por otra parte y como destacan ARMENTERAS et al., "igualmente esencial para los ecólogos porque permite «traducir» la funcionalidad y complejidad del ecosistema a herramientas para su uso en ámbitos ajenos al de la ciencia de la ecología, como por ejemplo en procesos participativos, en la toma de decisiones sobre recursos naturales y finalmente en el manejo del territorio"15.

\section{EL INTENTO DE AGRUPACIÓN DE LOS CONCEPTOS EN BASE A SU DIFERENTE ORIGEN Y FINALIDAD}

Existen de este modo conceptos generales que marcan o dejan su impronta en el enfoque global desde el que el Derecho acomete la protección y conservación ambiental. En este caso, sin duda, el de «riesgo» (ESTEVE) ${ }^{16}$ permea todos los

ordenada de los recursos para garantizar el aprovechamiento sostenible del patrimonio natural, en particular, de las especies y de los ecosistemas, su conservación, restauración y mejora y evitar la pérdida neta de biodiversidad" y d) ("La conservación y preservación de la variedad, singularidad y belleza de los ecosistemas naturales, de la diversidad geológica y del paisaje...").

${ }^{14}$ D. Armenteras / T. M., GonzÁlez / L. K. Vergara / F. J. LUQUe / N. RodríGUez / M. A. BoniLLA (2016), "Revisión del concepto de ecosistema como «unidad de la naturaleza» 80 años después de su formulación", Ecosistemas-Revista Científica de Ecología y Medio Ambiente, vol. 25, núm. 1 , p. 87.

15 lbídem, p. 88.

${ }^{16}$ Una magnífica síntesis sobre sus reflexiones sobre este concepto que ha abordado el autor de forma más extensa en las monografías ya reseñadas en José ESTEVE PARDO (2006), "EI Derecho del medio ambiente como derecho de decisión y gestión de riesgos", Revista electrónica de Derecho-Universidad de La Rioja (Redur), vol. 4, pp. 7-16.

Especialmente significativa resulta la constatación de ESTEVE del fenómeno de retracción o, como también lo denomina, de encogimiento del Derecho como consecuencia de la complejidad tecnológica y de las incertidumbres sobre los riesgos que genera: "el Derecho medioambiental es un Derecho que se está convirtiendo, si no lo remediamos, en un Derecho anómico, un 
modernos estudios sobre la materia ${ }^{17}$. Otros, de cuña más bien sociológica, serían los de «vulnerabilidad», el de «globalización», este último, también con un fuerte componente economicista y, el proveniente de la ciencia política, de "gobernanza»18. Otros de estos conceptos tienen un carácter puramente técnico, ligados a la lucha contra las emisiones, como el de «mejor técnica disponible» o el de «valores genéricos de referencia»; también otros se han elaborado para medir el impacto ambiental de determinados usos de los recursos naturales como el de «huella ecológica» ${ }^{19}$.

Derecho sin normas, un Derecho en el que las normas jurídicas tienen un protagonismo estrictamente formal, procedimental" (Ibídem, p. 16).

17 Un ejemplo significativo, por citar un ámbito especialmente discutido y donde las empresas productoras más intensamente defienden de forma convincente por diversos medios la bondad de sus productos, se produce en relación a los potenciales efectos tóxicos de los plaguicidas en relación a los cuales es necesario analizar e identificar su potencial de riesgo adverso hacia el medio ambiente y los seres humanos. Cfr. Blanca SORO MATEO (2018), Derecho de los pesticidas, Tirant Lo Blanc, Valencia.

En relación específica a los riesgos de estos productos en relación a las aguas subterráneas me ocupé en Santiago M. ÁlvaREZ CARREÑo (2011), "Actividad agrícola y contaminación de aguas subterráneas: régimen jurídico", en Antonio EMBID IRUJO (Dir.), Agua y agricultura, Thomson/Civitas, Cizur Menor, pp. 215-281.

La reciente STJUE (Tribunal General) dictada en los asuntos T-716/14 Anthony C.Tweedale / Autoridad Europea de Seguridad Alimentaria (EFSA) y T-329/17 Hautala y otros/EFSA se anulan, en atención al principio de precaución ante los riesgos que presenta, las resoluciones de la EFSA por las que se deniega el acceso a los estudios de toxicidad y de carcinogenicidad de la sustancia activa glifosato.

${ }^{18}$ Sobre la gobernanza medioambiental en relación específica a la gestión de espacios naturales protegidos vid. José Luis BERMEJO LATRE (2016), "La participación del público en la protección de la biodiversidad", en Gerardo GARCíA-ÁlvaREZ (Ed.), Instrumentos territoriales para la protección de la Biodiversidad, Monografías de la Revista Aragonesa de Administración Pública, XVI, 2016, pp. 151-176.

19 Una aplicación específica de este es el de «huella hídrica» que sirve para describir las relaciones entre el agua consumida de forma directa o indirecta y los procesos de contaminación hídrica. Como nos recuerdan IVANOVA/ SARMIENTO/ DOMínGUEZ, en los años noventa del siglo anterior Arjen HOEKSTRA, de UNESCO-IHE, introdujo el término huella hídrica que fue empleado para evaluar las dinámicas del agua como indicador de presión sobre el recurso hídrico. El término se entiende como "el uso de agua que tiene en cuenta tanto el uso directo como indirecto por parte de un consumidor o productor" (HOEKSTRA, 2004). Por su parte, la huella hídrica se divide en tres tipos en función del origen de las aguas usadas para satisfacer las demandas del recurso hídrico para uno u otro fin. Así, la huella hídrica azul se relaciona con el uso de las aguas superficiales y subterráneas, la huella hídrica verde se refiere al uso de las aguas provenientes de las lluvias, y la huella hídrica gris hace referencia al volumen del agua requerido para neutralizar la concentración de los contaminantes introducidos a uno u otro cuerpo hídrico ( $\mathrm{Y}$. IVANOVA / A. SARMiento LóPEZ / E. A. Domínguez CAlle (2016), "Evaluación de la huella hídrica de la ciudad de Bogotá como una herramienta de la gestión del agua en el área urbana", en D. A. CARDONA ZeA / I. RESTREPO TARQUINo (Comp.), Manejo del riesgo en la gestión del agua. Retos ante los riesgos ambientales en el ciclo del agua, justicia ambiental y conflictos, Programa Editorial Universidad del Valle, Cali, p. 92). 
Muchos de ellos viene determinados por la necesidad de superar los fundamentos de una teoría económica clásica de ortodoxia liberal que carece de instrumentos para interiorizar los costes ambientales, de modo que el agente económico pague por la contaminación o daño ambiental que genera, incorporando así esta variable a sus costes de producción ${ }^{20}$. Surgen así conceptos tan esenciales para el Derecho ambiental como el de «desarrollo sostenible» 0 «sostenibilidad» 21 .

Más modernamente, inciden en este esfuerzo de trascender un modelo económico agresivamente capitalista, los de «economía circular» —al que dedica su contribución a este monográfico el prof. René SANTAMARÍA ARINAS ${ }^{22}$ , la «economía del bien común»o, en fin, la «economía verde»23 e, incluso, la «bioeconomía sostenible» 24 . En realidad, este esfuerzo por conseguir

20 Este nuevo modelo económico se posibilita a partir de la elaboración del concepto de «externalidades negativas», alumbrado a nivel teórico en 1920 por el economista Albert Cecil PIGOU en The Economics of Welfare. Como nos recuerda de manera magistral LOZANO, "la ciencia económica convencional había ignorado, hasta mediados del siglo pasado, la variable ambiental en la toma de decisiones, dado que la naturaleza parecía un proveedor inagotable de recursos naturales y un receptor sin fondo de emisiones y vertidos contaminantes. Pero a medida que los bienes comunes 0 «libres» empezaron a emitir señales de escasez o deterioro, cada vez más acusadas, científicamente contrastadas y perceptibles (contaminación de ríos y acuíferos; vertidos y emisiones nocivas para la salud, acelerada pérdida de biodiversidad y un largo etcétera), se hizo evidente la necesidad de cambiar de modelo" (LOZANO, "Derecho ambiental...", cit., p. 415).

La concesión del Premio Nobel de Economía en 2018 al economista norteamericano William D. NORDHAUS puede suponer un cambio de inflexión en cuanto se reconoce a este máximo nivel su esfuerzo de integración del cambio climático en los modelos económicos, en lo que se conoce como "Economía del Clima".

21 Cfr. José Francisco Alenza García (2010), "Desarrollo sostenible", en José Antonio SANTAMARÍA PAStOR, J. A. (dir.), Los principios jurídicos del Derecho Administrativo, La Ley, Madrid, pp. 1387-1426.

22 Vid. infra en este mismo número monográfico.

23 PNUMA (2011), Hacia una economía verde. Guía para el desarrollo sostenible y la erradicación de la pobreza.

Accesible en < http://sostenibilidadyprogreso.org/files/entradas/hacia-una-economia-verde.pdf> [Último acceso: 17/04/2019].

${ }^{24}$ En el Programa General de acción de la Unión en materia de Medio Ambiente hasta 2020, aprobado por la Decisión 1386/2013/UE, del Parlamento y del Consejo podemos leer: "en los últimos años, el empleo en los sectores de las tecnologías y servicios medioambientales en la Unión ha crecido en torno al 3 por ciento anual. El mercado mundial para las ecoindustrias se estima en, al menos, un billón de euros, y se prevé que en los próximos diez años llegue casi a duplicarse. Las empresas europeas ya son líderes mundiales en reciclado y eficiencia energética, y hay que estimularlas para que saquen provecho del aumento de la demanda en el mundo, con el apoyo del Plan de Acción sobre Ecoinnovación. Por ejemplo, se espera que el sector europeo 
contabilizar los efectos positivos o negativos de determinadas actividades 0 decisiones de consumo están detrás de muchos de estos nuevos conceptos que pueblan estudios e informes, que penetran los textos de soft law de instituciones internacionales y que, en esa deriva, acaban incorporándose a textos propiamente normativos o decisiones jurisprudenciales. La doctrina, en cualquier caso, está atenta a conceptos como el de «agua virtual», el de «servicios ecosistémicos» ${ }^{25}$, el de «soluciones basadas en la naturaleza» ${ }^{26}$ o, en fin, el de «capital natural».

Otra cuestión que ha generado aportes conceptuales con repercusión jurídica son los derivados de avances tecnológicos que plantean retos a los que el Derecho debe atender. Así, en su momento ocurrió con la «biomasa» (MARTíN MATEO), más recientemente con la técnica de «fracking» 27 o todo lo que por ser relativo al empleo de nuevas tecnologías recibe el calificativo de «smart» 28 .

de las energías renovables cree, por sí solo, más de 400.000 puestos de trabajo de aquí a 2020. Una bioeconomía sostenible también puede contribuir a un crecimiento inteligente y verde en Europa y, a la vez, beneficiarse de las mejoras de la eficiencia en el uso de recursos" (cito por LOZANO, cit., p. 413, n. 6).

${ }^{25}$ Cfr. M. MonTeduro (2013), "Environmental Law and Agroecology. Transdisciplinary Approach to Public Ecosystem Services as a New Challenge for Environmental Legal Doctrine", European Energy and Environmental Law Review, vol. 22, Issue 1, pp. 2-11; K. MERTENS / A. CLIQUET / B. BANHEUSDEN (2012), "Ecosystem Services: What's in it for a Lawyer", Energy \& Environmental Law Review, vol. 21, pp. 31 y ss.

${ }^{26}$ En el Informe Mundial de las Naciones Unidas sobre el Desarrollo de los Recursos Hídricos de 2018 se recoge que las soluciones basadas en la naturaleza "están inspiradas y respaldadas por la naturaleza y utilizan o imitan los procesos naturales para contribuir a la gestión mejorada del agua. Una solución basada en la naturaleza puede implicar la conservación o rehabilitación de los ecosistemas naturales y/o la mejora o creación de procesos naturales en ecosistemas modificados o artificiales". Cfr. S. PeRALEs MOMPARLER, Soluciones basadas en la naturaleza para la gestión del ciclo urbano del agua. Accesible en < https://www.camarazaragoza.com/wpcontent/uploads/2018/11/Ponencia-Sara-Perales.pdf> [Último acceso: 17/04/2019].

27 Vid. Germán Valencia Martín, G./ Juan Rosa MoReno (dir.) (2016), Derecho y "Fracking", Thomson Reuters/Aranzadi, Cizur Menor.

${ }^{28}$ Cfr. Rubén MARTíNEZ GUTIÉRREZ (2017), "El impacto de las «smart cities» en la tutela ambiental y en la planificación urbana", en José Luis PIÑAR MAÑAS (dir.), Smart Cities: derecho y técnica para una ciudad más habitable, pp. 53-72.

En materia de aguas se habla de "smartwater" para aludir a soluciones tecnológicas para el monitoreo de la calidad del agua que están ganado importancia con el avance de las tecnologías de la comunicación. Para una visión detallada de las últimas innovaciones tecnológicas en este campo con propuestas de sistemas de control menos costosos y complejos vid. S. GEETHA / S. GOUTHAMI (2017), "Internet of things enabled real time water quality monitoring system", Smart Water-International Journal for @qua, 2:1. 
Otro grupo conceptual deriva del que ha devenido, sin duda, en concepto clave y central que domina todo el discurso ambiental y centra todas las preocupaciones políticas y jurídicas, es decir, el «cambio climático» 29 . De él, a su vez, derivan los de «refugiado climático» ${ }^{30}$, «justicia climática» ${ }^{31} \mathrm{o}$, incluso, el que define una nueva edad climática, el de «antropoceno»32. En elpresente número monográfico la profesora Marta TORRE-SCHAUB analiza esta dimensión conceptual del fenómeno que, parafraseando a Naomi KLEIN, lo cambia todo ${ }^{33}$.

${ }^{29}$ Muy oportunamente el primer monógráfico de esta Revista Catalana de Dret Ambiental (Vol.
9, Núm. 2. 2018) se dedica precisamente a este tema. Accesible en
$<$ <https://revistes.urv.cat/index.php/rcda/issue/view/147> [Último acceso: 17/04/2019].

30 Según los autores que han definido este concepto, los refugiados ambientales o refugiados climáticos se definen como "aquellos individuos que se han visto forzados a dejar su «ambiente natural'", de forma temporal o permanente, debido a un marcado trastorno ambiental, ya sea a causa de peligros naturales y/o provocado por la actividad humana, poniendo en peligro su existencia y/o afectando seriamente su calidad de vida". Cfr. Susana BORRÀs PERTINAT (2006), "Refugiados ambientales: el nuevo desafío del Derecho internacional del medio ambiente", Revista de Derecho, vol. 19, núm. 2, pp. 85-108.

La necesidad de encontrar un concepto apropiado para esta realidad ligada a la vulnerabilidad especial de determinadas poblaciones o grupos de personas la encontramos ya en N. MYERS (1995), Environmental Refugees, Climate Institute of Washington D. C., 1995, p. 17 y ss.

31 Vid. Marta TORRE-SCHAUB, "Le contentieux climatique, quel apport en termes de droits" in Colloque annuel de la SFDE, Aix-en-Provence, 30 juin 2017 y "Justice et justiciabilité climatique", Ecole d'été Autour de $2^{\circ}$ Université de Grenoble, à Autrans du 12 au 16 juin 2017.

32 SANZ LARRUGA nos recuerda que el nombre de "Antropoceno" había sido popularizado en 2000 por Paul Crutzen, Premio Nobel de Química, para significar que nos encontramos en una nueva etapa caracterizada por el impacto de las actividades humanas sobre los procesos ecológicos del Planeta. En realidad, hace ya mucho tiempo que se venía hablando de la capacidad del ser humano para incidir profundamente sobre la geografía física de la Tierra. Así, por ejemplo, en la pionera obra "Man and Nature" (1864) del diplomático norteamericano George Perkins Marsh, denunciando los degradantes efectos antrópicos sobre el medio ambiente... Mucho más reciente, en la primera decena del presente siglo XXI, las muy prestigiosa "Sociedad Geológica de Londres" y la "Comisión Internacional de Estratigrafía" han admitido e, incluso, definido esta nueva etapa geológica. Esta posición sobre la nueva época geológica promovida por los seres humanos no ha estado exenta de voces críticas para quienes esta novedad es más una declaración política que una propuesta científica. Vid. Javier SANZ LARRUGA, “¿Bienvenidos al Antropoceno?" en su blog <http://blogs.lavozdegalicia.es> [Último acceso: 17/04/2019].

En la doctrina iusfilosófica vid. Teresa Vicente GimÉnEZ/ CARLos Berzosa Alonso-MarTínez (coord.) (2016), Justicia ecológica en la era del antropoceno, Trotta, Madrid.

33 Entre otras cosas, la determinación de la naturaleza antrópica de los cambios en el clima ha llevado a su extremo la tensión subyacente entre la ciencia y los científicos y la política. Vid. Duncan FRENCH y Benjamin PONTIN (2016), "The science of climate change: a legal perspective on the IPCC", en Daniel A. Farber / Sho Sato (editores), Climate change law, Elgar Encyclopedia of Environmental Law, vol. 1, Edward Elgar, Cheltenham, pp. 9-19. 
En fin muchos otros conceptos - «umbral»34, «resiliencia», «ecosistémico», «holístico», «aquitorio»35...- que van apareciendo en la doctrina jurídica y en los textos legales y resoluciones judiciales y que atestiguan ese continuo trasiego conceptual entre las ciencias de la naturaleza y las ciencias humanas hacia el Derecho ambiental, donde adoptan perfiles singulares, a veces, alejados de la ciencia matriz donde se forjaron.

\section{LA INCERTIDUMBRE CIENTÍFICA COMO FUENTE DE DEBILIDAD DEL DERECHO AMBIENTAL: SU REFLEJO EN LA JURISPRUDENCIA CONSTITUCIONAL ESPAÑOLA}

Ahora bien, los niveles de incertidumbre científica y de controversia conceptual que se producen de modo natural en el mundo de la investigación científica donde resulta connatural la existencia de un fuerte nivel de debate y de crítica entre posturas contradictorias 0 , por lo menos, divergentes - constituye sin embargo una fuente de debilidad para el Derecho ambiental que, obligado por la lógica jurídica orientada de modo finalista a permitir la resolución práctica de un conflicto de intereses en el ámbito de lo social, debe optar por acoger en su seno o, por el contrario, rechazar la recepción normativa de conceptos o principios sometidos al referido debate científico.

De esa situación de dependencia y de duda sobre el grado de fiabilidad y certeza del conocimiento científico alcanzado en determinado sector o materia sacan partido en ocasiones aquellas voces contrarias a una aplicación más exigente y extensiva de las normas protectoras del medio ambiente. En efecto, la importancia y funcionalidad de los conceptos científicos que acaban finalmente incorporados a los textos legales en normas ambientales también reside en su capacidad de resistencia frente a las posturas escépticas con el paradigma ambientalista que tanta difusión y aceptación social en determinados ámbitos

\footnotetext{
${ }^{34}$ Un análisis de este concepto que en el sentido de lo que se quiere enfatizar en esta introducción en L. PEYEN (2014), "Essai d'une approche épistémologique du seuil en droit de l'environnement", en MILON/SAMSON, cit., pp. 133-152.

35 Teresa PAREJo NAVAJAS (2012), "La custodia del aquitorio: participación ciudadana en la ordenación física de usos sobre los espacios marítimos", en Estanislao ARANA GARCía/ JAVIER SANZ LARRUGA, J./ Asensio NAVARRO ORTEGA (coord.), La ordenación jurídica del medio marino en España: estudios sobre la Ley 41/2010, de Protección del medio marino, pp. 647-682.
} 
tienen $^{36}$ y que encuentran modernamente cobijo de manera organizada en partidos conservadores de ideología extrema, nostálgicos incluso de los regímenes atroces surgidos en la Europa continental en los años 30 del pasado siglo $^{37}$.

También, de modo más larvado, donde no se trata tanto de negar la veracidad del consenso científico alcanzado sobre la necesidad de actuar de manera regulatoria para impedir o limitar los efectos perjudiciales sobre el medio ambiente de determinado sector de actividad sino de retrasar todo lo posible la adopción por los poderes públicos de dichas decisiones para ganar tiempo y ventajas competitivas para un posicionamiento aventajado en el futuro mercado emergente - una nueva tecnología, una nueva fuente de energía, un nuevo producto... - que sustituya al anterior devenido obsoleto por mor de sus comprobados impactos negativos ambientales. Es en este campo donde más bien podría situarse el conocido greenwashing de determinadas empresas en su intento de aplazar todo lo posible la completa descarbonización del proceso productivo ${ }^{38}$ o las labores de lobby cerca de los gobiernos para postergar las medidas exigidas por la lucha contra el cambio climático ${ }^{39}$. En muchas ocasiones, estos argumentos provenientes de los sectores afectados — vestidos para la ocasión con argumentos expertos que logran llevar a la zona de incertidumbre al responsable público competente- permean la labor

\footnotetext{
36 Piénsese en el libro de Michel CRICHTON Estado de miedo o en el de LOMBORG El ambientalista escéptico, por citar sólo dos de los más populares autores críticos con los postulados del movimiento ecologista e, incluso, con las creencias científicas admitidas como más verosímiles o razonables en la actualidad. Se podrían también rastrear las múltiples webs y publicaciones en diversos formatos que propagan versiones negacionistas del cambio climático con imputaciones de conspiración de oscuros intereses ecologistas que, no por rayar en lo paranoide, disfrutan de un mayor auditorio del que podríamos llegar a pensar.

37 Cfr. el estudio y análisis de estas estrategias en S. Stella SchalleR/ A. CARIUS (2019), Convenient Truths Mapping climate agendas of right-wing populist parties in Europe, adelphi consult GmbH, Berlin

38 Vid. sobre este concepto The Guardian, "The troubling evolution of corporate greenwashing". Accesible en <https://www.theguardian.com/sustainable-business/2016/aug/20/greenwashingenvironmentalism-lies-companies> [último acceso: 28/03/2019].

39 Cfr. el informe de INFLUENCEMAP, Big Oils's Real Agenda on Climate Change. Accesible en $<$ https://influencemap.org/report/Climate-Lobbying-by-the-Fossil-Fuel-Sector> [Último acceso: 28/03/2019].

En él se asegura que ExxonMobiL, Shell, CheVron, British Petroleum (BP) y Total se han gastado desde los Acuerdos de París de 2015 más de 1.000 millones de dólares en estrategias de lobby, que han hecho coincidir con campañas de lavado de imagen a favor de las energías limpias.
} 
administrativa y acaban cristalizando en reformas regresivas de la legislación ambiental ${ }^{40}$.

Precisamente, el principio de no regresión, definido por AMAYA ARIAS como la "limitación a los poderes públicos de disminuir o afectar de manera significativa el nivel de protección ambiental alcanzado, salvo que esté absoluta y debidamente justificado" 41 —aceptado ya de manera general como un principio del Derecho ambiental ${ }^{42}$ — vendría a impedir estas modificaciones de los niveles o estándares alcanzados de protección ambiental excepto que los avances en el conocimiento científico o las innovaciones tecnológicas hicieran compatible la reducción del arsenal normativo con la mejora o, por lo menos, el mantenimiento del nivel de protección material anterior ${ }^{43}$.

No lo considera así todavía nuestro Tribunal Constitucional que, en su Sentencia del Pleno, de 5 de noviembre de 2015, resuelve un recurso interpuesto contra los artículos de la Ley 2/2013, de 29 de mayo, de protección y uso sostenible del litoral y de modificación de la Ley 22/1988, de 28 de julio, de costas donde se valoró si la modificación operada provocaba o no un impacto negativo sobre la conservación del medio ambiente. En ella afirma el TC que (...) En particular, el deber de conservación que incumbe a los poderes públicos tiene una dimensión,

\begin{abstract}
${ }^{40}$ El rastreador de regresiones normativas en materia ambiental («Regulatory Rollback Tracker») de la Universidad de Harvard en EEUU acredita que la actual Administración Trump ha modificado o está en proceso de hacerlo unas 80 regulaciones ambientales que reducen los estándares de protección ambiental. Estas regresiones ambientales podrían causar, según documentan en estudios investigadores de esa misma Universidad, al menos 80.000 muertes adicionales cada década y provocar problemas respiratorios a más de un millón de personas. Vid. THE NEW YORK TIMES, "78 Environmental Rules on the Way Out Under Trump". Accesible en $<$ https://www.nytimes.com/interactive/2017/10/05/climate/trump-environment-rulesreversed.html> [Último acceso: 29/03/2019].

${ }^{41}$ Ángela María AmAYA ARIAS (2016), El principio de no regresión en el Derecho ambiental, lustel, Madrid. En concreto, la relación entre la ciencia y el Derecho ambiental en pp. 334 y ss.

42 Defiende LÓPEZ RAMÓN que, en España, de las obligaciones derivadas para los poderes públicos en el art. 45. 2 CE orientadas a proteger, mejorar y restaurar el medio ambiente se deduce la obligación para el legislador de no incurrir en una regresión en la protección del medio ambiente. Cfr. Fernando LOPEZ RAMON (2005), "L'environnement dans la Constitution espagnole", Revue juridique de l'environnement, núm. especial, pp. 53-62. Se ocupa también de estas cuestiones en Fernando LÓPEZ RAMÓN (2016), "La aceptación legislativa del principio de no regresión ambiental en Francia”, Revista de Administración Pública, núm. 201, pp. 269-277.
\end{abstract}

43 Vid. Blanca LOZANO CUTANDA (2012), "Principio Standstill versus Discrecionalidad Administrativa", en Eduardo GARCíA DE ENTERRÍA (coord.), Administración y Justicia: un análisis jurisprudencial. Liber amicorum Tomás Ramón Fernández, Madrid. 
la de no propiciar la destrucción o degradación del medio ambiente, que no consentiría la adopción de medidas, carentes de justificación objetiva, de tal calibre que supusieran un patente retroceso en el grado de protección que se ha alcanzado tras décadas de intervención tuitiva. Esta dimensión inevitablemente evoca la idea de "no regresión», aunque los conceptos que estamos aquí contrastando no admiten una identificación mecánica, pues es también de notar que el deber constitucional se proyecta sobre el medio físico, en tanto que el principio de no regresión se predica del ordenamiento jurídico. En términos constitucionales, esta relevante diferencia significa que la norma no es intangible, y que por tanto la apreciación del potencial impacto negativo de su modificación sobre la conservación del medio ambiente requiere una cuidadosa ponderación, en la que, como uno más entre otros factores, habrá de tomarse en consideración la regulación preexistente (...) ${ }^{44}$. No se comparte esa disociación escolástica entre un deber constitucional de protección y conservación del medio ambiente que se proyectaría sobre el medio físico frente a las consecuencias deducibles del principio de no regresión que, en la interpretación del TC, se limitarían a aplicarse en el ámbito de lo jurídico ${ }^{45}$.

Se pueden detectar — siguiendo el magnífico análisis de NoGUEIRA - tres manifestaciones principales del estrechamiento de la protección ambiental por obra de una jurisprudencia del TC español que se amolda al signo desregulatorio de los tiempos y que se muestra absolutamente deferente con el legislador y, más allá, con el Gobierno central, rebajando la consideración jurídica y constitucional de las cuestiones ambientales: “... por un lado la resistencia del TC a acoger los parámetros interpretativos que se derivan de los tratados

\footnotetext{
${ }^{44}$ Mantiene esta doctrina en la STC 132/2017, de 14 noviembre, por la que se desestima el recurso de inconstitucionalidad promovido por más de 50 diputados del Grupo Parlamentario Confederal de Unidos Podemos-En Comú Podem-En Marea en el Congreso de los Diputados contra la Ley de Asturias 2/2017, de 24 de marzo, de segunda modificación de la Ley 3/2004, de 23 de noviembre, de montes y ordenación forestal. EI TC obliga a superar el que denomina test de reversibilidad de las medidas reductoras del nivel de protección ambiental, pero no el de no regresión (...) máxime cuando, como aquí es el caso, no hay unanimidad técnica sobre el impacto del pastoreo y se carece de una base epistemológica unívoca e irrefutable para hablar con absoluta certeza de regresión o de minoración de la protección medioambiental.

45 Cfr. Álvarez Carreño, S. M. (2018), "Las regresiones del derecho ambiental como causa de aumento del riesgo sobre grupos vulnerables", lus et Scientia. Revista electrónica de Derecho y Ciencia, vol. 4, núm. 2 (Epigenética, Transhumanismo y Grupos vulnerables), pp. 31- 49; y "La evolución regresiva de la política y del derecho ambiental en España", Revista Aranzadi de Derecho Ambiental, núm. 41, pp. 15-40.
} 
internacionales y sentencias ex art. 10. 2 CE y a acompasar su jurisprudencia con formulaciones más innovadoras en términos de reforzamiento de la protección ambiental. En segundo término, el rechazo a extraer del art. $45 \mathrm{CE}$ elementos interpretativos para la solución material de controversias constitucionales, aunque estas surjan de un conflicto competencial. En tercero, y ligado a la anterior, dar primacía con carácter creciente a títulos competenciales «desarrollistas» frente a la protección del medio ambiente en una tensión Estado/Comunidades autónomas que también es, en muchos casos, productivismo/tutela ambiental"46.

\section{FINAL: LA NECESIDAD DE UN DIÁLOGO FLUIDO Y FRUCTÍFERO ENTRE LOS DIFERENTES SABERES QUE CONFLUYEN EN LA DETERMINACIÓN DEL CONTENIDO DE LAS NORMAS AMBIENTALES}

Para un aquilatamiento más ajustado de las complejas interacciones entre lo científico, lo jurídico y lo político serían necesarios profundos conocimientos de carácter interdisciplinar que permitieran relacionar los resultados de la investigación sobre historia de la ciencia con los de epistemología, ecología y, todo ello, a la luz de un profundo dominio del derecho positivo y de su interpretación jurisprudencial. La contraposición dialéctica del Derecho ambiental como rama del ordenamiento jurídico con la exigencia derivada de la racionalidad y objetividad científica permitirían conceptualizar y delimitar los aportes correspondientes a estos conocimientos múltiples y plurales. Si estuviéramos en condiciones de llevar a cabo un esfuerzo tal, posiblemente acabaríamos concluyendo que el brillo y, a la vez, la relativa indigencia del Derecho ambiental derivan precisamente de ese intento por encontrar el valor de lo justo en campos donde reinan la indeterminación, la falta de certezas absolutas y la complejidad de la tensa interacción de poderosos intereses contrapuestos ${ }^{47}$. Para NAIM-

\footnotetext{
46 Alba NogueiRA LÓPEZ (2018), "El demediado derecho a un medio ambiente adecuado", en PENDÁs, B. (dir.), España constitucional (1978-2018), t. III - Trayectorias y perspectivas, Centro de Estudios Políticos y Constitucionales, Madrid, p. 2472). Como concluye la autora precitada, "la existencia de argumentaciones contradictorias para dar prevalencia a los títulos noambientales revela la necesidad de que se realice una reflexión unitaria sobre el significado del art. 45 CE y el mandato de protección constitucional que representa" (Ibídem, p. 2482).
}

47 NAIM-GeSBERT, Dimension scientifiques..., cito por BORN/SADELEER, cit., p. 557. 
GeSBERT se detecta así una verdadera tensión entre la «verdad científica» que deriva del orden de las cosas y la «verdad jurídica» que emana del ordenamiento jurídico. Para este autor, el ordenamiento ambiental persigue una doble finalidad: la protección eficaz del medio y la remodelación de la sociedad en el sentido de los imperativos ecológicos. El pluralismo de verdades se convierte aquí en una verdadera «política de la ciencia», es decir, una política que se adscribe a un campo democrático. El derecho se cae de su pedestal. No siendo ya portador de un discurso único de la verdad, cumple más bien una función de mediador entre la ciencia y la cultura en un estado permanente de redefinición.

Esta característica determina también que el grado de aplicación efectiva y real de las prescripciones jurídico-ambientales —la desuetudo, por emplear la afortunada expresión de JORDANO FRAGA, constituye el verdadero talón de AQUILES de la disciplina - venga determinado por el nivel de aceptación general y por el apoyo social que reciban los mandatos jurídico ambientales. Para el caso paradigmático de la determinación del origen antrópico como causa del proceso de calentamiento global del clima del planeta, destacan Cook et alt. que la percepción de la sociedad sobre el grado de consenso científico sobre este origen constituye un elemento imprescindible para el apoyo a las políticas de mitigación de los efectos del cambio climático. Sin embargo, existe una diferencia significativa entre la percepción pública y la realidad, cuando el $57 \%$ de la sociedad de los EEUU o bien no está de acuerdo o sencillamente no sabe que los científicos coinciden de manera abrumadora en que el clima de la tierra se está calentando debido a la actividad humana ${ }^{48}$. En esta misma línea de

48 J. CoOK / D. Nuccitelli / S. A. GReEN / M. Richardson / B. WinKLER / R. PAinting / R. WAY / P. JACOBS / A. SKUCE (2013), "Quantifying the consensus on anthropogenic global warming in the scientific literatura", Environmental Research Letters, vol. 8, p. 6.

Contribuyen a esta distancia entre la realidad y la percepción social las campañas diseñadas para confundir al público sobre el grado de acuerdo entre los científicos del clima. Así, estos autores recuerdan que, en 1991, la Western Fuels Association llevó a cabo una campaña de comunicación por valor de 510 mil dólares que tenía como objetivo principal "devolver el calentamiento global a la categoría de teoría, no de hecho". Una estrategia clave utilizada era dar la impresión de que existía un debate científico activo mediante la utilización de científicos disidentes como portavoces. La situación se exacerba en el tratamiento mediático cuando el protocolo exige otorgar la misma atención a las partes opuestas lo que permite en la práctica a una minoría amplificar sus puntos de vista. Mientras que la situación parece haber mejorado en los medios de comunicación de prestigio tanto en el Reino Unido como en los EEUU, la prensa amarilla del Reino Unido no había mostrado ninguna mejoría en el tratamiento de estas cuestiones desde el 2000 hasta el 2006 (Ibídem). 
pensamiento, destaca LOZANO que "la sociedad utiliza el derecho para dar una respuesta a los retos y dilemas de cada tiempo histórico. El problema que se aborda con el derecho ambiental es, a decir de los científicos, el de la desestabilización antrópica de la biosfera: como resultado de las fuerzas motrices activadas por la revolución industrial (crecimiento económico, explosión demográfica -en el siglo XX la población humana se triplicó y los demógrafos de Naciones Unidas prevén que alcance los 9.600 millones de personas a finales del presente siglo, uso masivo de las energías fósiles, consumo de masas-), una infinidad de precisiones e impactos ambientales con efecto acumulativo y sinérgico han provocado la desestabilización de las funciones de soporte de la biosfera, y la comunidad científica insiste en que existe el riesgo cierto de un cambio irreversible" 49 .

Los estudios de las profesoras TORRE-SCHAUB sobre "cambio climático", de NOGUEIRA sobre «huella ecológica», y REVUELTA sobre «mejor tecnología disponible» y de los profesores ALENZA sobre «vulnerabilidad» y SANTAMARÍA sobre «economía circular» incluidos en este número monográfico - todas ellas contribuciones excelentes de excelentes investigadores-, muestran una línea de fuerza del Derecho ambiental que tiene que ver con la necesidad de un diálogo fluido y fructífero entre los diferentes saberes que confluyen en la determinación de sus prescripciones, en las que deben además quedar recogidos los acuerdos de base participativa y democrática sobre qué derecho ambiental quiere tener esta sociedad para garantizar el nivel protección de nuestro medio que se estime, en cada momento histórico, deseable o exigible.

Se busca enfatizar que la búsqueda de la racionalidad y de la objetividad en una disciplina jurídica como la ambiental tiene que tener en cuenta necesariamente los condicionantes provenientes de las otras ciencias que estudian el medio físico, sus componentes e interacciones, así como el factor social ${ }^{50}$. Siendo

\footnotetext{
49 LOZANO CUTANDA, “Derecho ambiental...", cit., p. 410.

50 Muy interesantes reflexiones sobre la compleja interacción entre riesgos, intereses económicos, incertidumbres científicas y la necesidad de tomar decisiones por los responsables políticos que eviten posibles daños en sociedades democráticas se obtienen de la lectura de EUROPEAN ENVIRONMENTAL AGENCY (EEA), Environmental Issue Report $n^{\circ}$ 22: Late lessons from early warnings: the precautionary principle 1896-2000, Office for Official Publications of the European Communities, Luxembourg, 2001.
} 
conscientes de que existe un debate arduo y complejo que contrapone la ciencia con el derecho y que los poderosos intereses que, en este preciso momento de la necesaria transición ecológica del sistema económico y social, pretenden cortar las alas al ambientalismo manejan ingentes recursos al servicio de este fin, en estas líneas simplemente se ha pretendido hacer una aportación a la disciplina jurídica ambiental, conscientes tanto de sus logros como de sus manifiestas carencias y limitaciones.

Desde una consideración disciplinar, estas reflexiones introductorias también caminan en la misma dirección de las que con carácter general para el Derecho administrativo expresa MARCHECO ACUÑA cuando requiere profundizar "en las relaciones interdisciplinares que permitan al Derecho administrativo construir sus categorías conceptuales y metodológicas con un mayor aporte de otras ciencias (políticas, económicas, de la Administración, sociología) o con soluciones de otros sistemas jurídicos (derecho comparado). Ello haría posible una ciencia del Derecho administrativo mucho más amplia y contextualizada, en tanto se ocuparía no ya sólo de los aspectos puramente «legales» de la actuación administrativa o de la defensa de los administrados frente a las intromisiones ilegítimas de la Administración, sino también de la consideración de los efectos y consecuencias de dicha actuación, de la eficiencia, la calidad y las garantías de los resultados" 51 .

\section{BIBLIOGRAFÍA}

- Alenza García, J. F. (2010), "Desarrollo sostenible", en Santamaría Pastor, J. A. (dir.), Los principios jurídicos del Derecho Administrativo, La Ley, Madrid, pp. 1387-1426.

En él se pone de manifiesto que la aceptación por la sociedad de los riesgos derivados de actividades económicas requiere su participación en la toma de las decisiones que crean esos riesgos y el modo de gestionarlos. Una política adecuada en relación a los problemas ambientales exige, además de un conocimiento científico objetivo, que se expliciten las opciones éticas que están en juego en cada una de las decisiones.

51 Benjamín MARCHECO ACUÑA (2018), "Los desafíos de la ciencia del Derecho administrativo ante la globalización", Revista General de Derecho Administrativo, núm. 49, p. 23. 
- Álvarez Carreño, S. M. (2018), "La evolución regresiva de la política y del derecho ambiental en España", Revista Aranzadi de Derecho Ambiental, núm. 41 , pp. $15-40$.

--- (2018) "Las regresiones del derecho ambiental como causa de aumento del riesgo sobre grupos vulnerables", Ius et Scientia. Revista electrónica de Derecho y Ciencia, vol. 4, núm. 2 (Epigenética, Transhumanismo y Grupos vulnerables), pp. 31- 49.

--- (2011) "Actividad agrícola y contaminación de aguas subterráneas: régimen jurídico", en Embid IRUJo, A. (Dir.), Agua y agricultura, Thomson/Civitas, Cizur Menor, pp. 215-281.

--- (2010) "Los derechos privados sobre el agua en países de common law: reflexiones sobre su evolución y adaptación a las necesidades de cambio", en Betancor Rodríguez, A., "Nuevas tendencias en el Derecho ambiental", Revista General de Derecho Administrativo, núm. 25, octubre 2010

- AmAYA ARIAS, A. M. (2016), El principio de no regresión en el Derecho ambiental, lustel, Madrid.

- Arana GarcíA, E. "Organización administrativa de la ciencia y tecnología en España", Documentación Administrativa, núms. 265-266 (enero-agosto 2003) Derecho Administrativo. Ciencia y Nuevas tecnologías, pp. 17-50.

- Armenteras, D./ González, T. M./Vergara, L. K./Luque, F. J., RodríGuez, N./Bonilla, M. A. (2016), "Revisión del concepto de ecosistema como «unidad de la naturaleza» 80 años después de su formulación", Ecosistemas-Revista Científica de Ecología y Medio Ambiente, vol. 25, núm. 1, pp. 83-89.

- BeRMEJo LATRE, J. L. (2016), "La participación del público en la protección de la biodiversidad", en GarcíA-ÁLVAREZ, G. (Ed.), Instrumentos territoriales para la protección de la Biodiversidad, Monografías de la Revista Aragonesa de Administración Pública, XVI, 2016, pp. 151-176.

- Betancor Rodríguez, A. (2010), "Nuevas tendencias en el Derecho ambiental", Revista General de Derecho Administrativo, núm. 25, octubre 2010.

--- (2014) Derecho ambiental, La Ley, Madrid. 
- Born, Ch.-H. / De Sadeleer, N. (2001), nota bibliográfica al libro de NaimGesbert, E., Les dimensions scientifiques du droit de l'environnement Contribution à l'étude des rapports de la science et du droit, en Revue Juridique de l'Environnement, núm. 3, pp. 555-557.

- Borràs Pertinat, S. (2006), "Refugiados ambientales: el nuevo desafío del Derecho internacional del medio ambiente", Revista de Derecho, vol. 19, núm. 2, pp. 85-108.

- Cierco Seira, C. (2004), "El principio de precaución: reflexiones sobre su contenido y alcance en los derechos comunitario y español", Revista de Administración Pública, núm. 163, pp. 73-126.

- Cook, J./ Nuccitelli, D./ Green, S. A./ Richardson, M./ Winkler, B. / Painting, R./ WaY, R./ JACOBS, P./ SKUCE, A. (2013), "Quantifying the consensus on anthropogenic global warming in the scientific literatura", Environmental Research Letters, vol. 8, pp.1-7.

- Crichton, M. (2010), Estado de miedo, Debolsillo-Grupo Plantea, Barcelona

- Doménech PAscual, G. (2006), Derechos fundamentales y riesgos tecnológicos: el derecho del ciudadano a ser protegido por los poderes públicos, Centro de Estudios Políticos y Constitucionales, Madrid

- Embid Tello, A. E. (2009), “El principio de precaución”, en SANTAMARÍA PAStor, J. A. (dir.), Los principios jurídicos del Derecho Administrativo, La Ley, Madrid, pp. 1221-1254.

- Esteve Pardo, J. (2009) El desconcierto de Leviatán. Política y derecho ante las incertidumbres de la ciencia, Marcial Pons, Madrid.

--- (2006), "El Derecho del medio ambiente como derecho de decisión y gestión de riesgos", Revista electrónica de Derecho-Universidad de La Rioja (Redur), vol. 4, pp. 7-16.

---- (2003), "Ciencia y Derecho ante los riesgos para la salud. Evaluación, decisión y gestión”, Documentación Administrativa, núm. 265-266, pp. 137-149. --- (1999), Técnica, riesgo y derecho. Tratamiento del riesgo tecnológico en el derecho ambiental 
- French, D. / Pontin, B. (2016), "The science of climate change: a legal perspective on the IPCC", en Daniel A. Farber/Sho Sato (editores), Climate change law-Elgar Encyclopedia of Environmental Law, vol. 1, Edward Elgar, Cheltenham, pp. 9-19.

- Geetha, S. / Gouthami, S. (2017), "Internet of things enabled real time water quality monitoring system", Smart Water_International Journal for @qua, 2:1.

- Ivanova, Y./ Sarmiento López, A./ Domínguez Calle, E. A. (2016), "Evaluación de la huella hídrica de la ciudad de Bogotá como una herramienta de la gestión del agua en el área urbana", Revista Ambiental —Aire, Agua, suelo, vol. 4, núm. 2, 2013, pp. 1-5 (reeditado en Cardona ZeA, D. A. / Restrepo Tarquino, I. (Comp.), Manejo del riesgo en la gestión del agua. Retos ante los riesgos ambientales en el ciclo del agua, justicia ambiental y conflictos, Programa Editorial Universidad del Valle, Cali).

- Jordano Fraga, J. (2003), "Ciencia, tecnología, medio ambiente y responsabilidad patrimonial de la Administración: en especial, los denominados riesgos del desarrollo", Documentación Administrativa, núms. 265-266 (eneroagosto 2003) -Derecho Administrativo. Ciencia y Nuevas tecnologías, pp. 237265.

- KLÄY, A./ ZIMmermanN, A. B./SCHNEIDER, F., "Rethinking science for sustainable development: Reflexive interaction for a paradigm transformation", Futures, vol. 65, 2015, pp. 72-85.

- Lomborg, B. (2003) El ambientalista escéptico, Espasa-Calpe,Madrid

- LÓPEZ RAMÓN, F., (2016), "La aceptación legislativa del principio de no regresión ambiental en Francia", Revista de Administración Pública, núm. 201, pp. 269277.

--- (2005), "L'environnement dans la Constitution espagnole", Revue juridique de l'environnement, núm. especial, pp. 53-62.

- LozANO CutANDA, B. (2016), "Derecho ambiental: algunas reflexiones desde el Derecho administrativo", Revista de Administración Pública, núm. 200, pp. 409438. 
--- (2012) "Principio Standstill versus Discrecionalidad Administrativa", en GARcíA DE ENTERRÍA, E. (coord.), Administración y Justicia: un análisis jurisprudencial. Liber amicorum Tomás Ramón Fernández, Madrid, pp. 1545-1574.

- Marcheco Acuña, B. (2018), "Los desafíos de la ciencia del Derecho administrativo ante la globalización", Revista General de Derecho Administrativo, núm. 49, pp. 1-26.

- Martín Mateo, R., (2017), "La revolución ambiental pendiente", Revista Aranzadi de Derecho Ambiental, núm. 38, pp. 21-41.

--- (2008) La verde energía de la biomasa, Thomson-Civitas, Madrid

--- (2001) "El avance científico: nuevos retos para el derecho sanitario" (DS Derecho y salud, vol. 9, núm. 1, enero-junio, pp. 21-26.

- Martínez GutiérRez, R. (2017), "El impacto de las «smart cities» en la tutela ambiental y en la planificación urbana", en PIÑAR MAÑAS, J. L. (dir.), Smart Cities: derecho y técnica para una ciudad más habitable, pp. 53-72.

- Mertens, K./ Cliquet, A. / Banheusden, B. (2012), "Ecosystem Services: What's in it for a Lawyer", Energy \& Environmental Law Review, vol. 21, pp. 31 y ss.

- MeYNer, A. (2014), "Le rôle des concepts dans la fondamentalisation du droit de l'environnement", en MILON, P./SAMSON, D., Révolution juridique, révolution scientfique. Vers une fondamentalisation du droit de l'environnement, Presses Universitaires d'Aix-Marseille (PUAM), Aix-en-Provence, pp. 113-131.

- Monteduro, M. (2013), "Environmental Law and Agroecology. Transdisciplinary Approach to Public Ecosystem Services as a New Challenge for Environmental Legal Doctrine", European Energy and Environmental Law Review, vol. 22, Issue 1, pp. 2-11.

- Montoro Chiner, M. J. (2003), "Seguridad jurídica, principio de cautela y comités científicos", Documentación Administrativa, Documentación Administrativa, núms. 265-266 (enero-agosto 2003) -Derecho Administrativo. Ciencia y Nuevas tecnologías, pp. 319-364.

- Moore, J.W./ Nowlan, L. / Olszynski, M./ JacoB, A.L./ Favaro, B./ Collins, L./ WILLIAMS DAVIDSON GLT.-L./ WEITZ, J. (2018), "Towards linking environmental law 
and science", FACETS - a multidisciplinary open access science journal, vol. 3, pp. $375-391$.

- MYERS, N. (1995), Environmental Refugees, Climate Institute of Washington D. C., 1995.

- Nogueira López, A. (2018), "El demediado derecho a un medio ambiente adecuado", en PENDÁs, B. (dir.), España constitucional (1978-2018), t. III Trayectorias y perspectivas, Centro de Estudios Políticos y Constitucionales, Madrid, pp. 2465-2482.

- OWen, D./ Noblet, C. (2015), "Interdisciplinary Research and Environmental Law", Ecology Law Quaterly, vol. 41, Issue 4, pp. 887-938.

- PAREJo Alfonso, L. (coord.) (2015), El derecho ante la innovación y los riesgos derivados del cambio climático, Tirant lo Blanch, Valencia.

- PAREJo NAVAJAS, T. (2012), "La custodia del aquitorio: participación ciudadana en la ordenación física de usos sobre los espacios marítimos", en ARANA GARCíA, E./ Sanz larruga, J./ Navarro Ortega, A. (coord.), La ordenación jurídica del medio marino en España: estudios sobre la Ley 41/2010, de Protección del medio marino, pp. 647-682.

- Peyen, L. (2014), “Essai d'une approche épistémologique du seuil en droit de l'environnement", en P. MILON/ D. SAMSON (coord.), Révolution juridique, Révolution scientifique. Vers une fondamentalisation $d u$ droit de l'environnement?, Presses Universitaires d'Aix-Marseille, Aix-en-Provence, pp. 133-152.

- Pomade, A. (2012), "Penser l'interdisciplinarité par l'internormativité. Illustration en droit de l'environnement", Revue interdisciplinaire d'études juridiques, 2012/1, vol. 68, pp. 85-106.

- SANZ LaRruga, J., “¿Bienvenidos al Antropoceno?”, en blog $<$ http://blogs.lavozdegalicia.es $>$.

- Soro Mateo, B. (2018), Derecho de los pesticidas, Tirant Lo Blanc, Valencia.

--- (2017), "Construyendo el principio de precaución", Revista Aragonesa de Administración Pública, núm. 49-50, pp. 87-151. 
- Stella Schaller, S./ Carius, A. (2019), Convenient Truths Mapping climate agendas of right-wing populist parties in Europe, adelphi consult $\mathrm{GmbH}$, Berlin

- TORRE-SchaUB, M. (2017), "Le contentieux climatique, quel apport en termes de droits", in Colloque annuel de la SFDE, Aix-en-Provence, 30 juin

--- (2017) “Justice et justiciabilité climatique", Ecole d'été Autour de $2^{\circ}$ Université de Grenoble, à Autrans, du 12 au 16 juin.

- Valencia Martín, G./ Rosa Moreno, J. (dir.) (2016), Derecho y "Fracking", Thomson Reuters/Aranzadi, Cizur Menor.

- Vicente Giménez, T./ Berzzosa Alonso-Martínez, C. (coord.) (2016), Justicia ecológica en la era del antropoceno, Trotta, Madrid. 